This article is licensed under the Creative Commons Attribution-NonCommercial 4.0 International License (CC BY-NC) (http://www.karger.com/Services/OpenAccessLicense). Usage and distribution for commercial purposes requires written permission.

\title{
Erythema Nodosum: A Manifestation of Salmonella Infection
}

\author{
Ali Hassan $^{a}$ Amna Alsaihati ${ }^{b}$ Malak Al Shammarib \\ Mohammed Sharroufna ${ }^{c}$ Haitham Alaithan ${ }^{a}$ Salman Aljubran ${ }^{d}$ \\ aDepartment of Internal Medicine, King Fahd Hospital of the University, Imam \\ Abdulrahman Bin Faisal University, Al-Khobar, Saudi Arabia; ${ }^{b}$ Department of Family and \\ Community Medicine, College of Medicine, Imam Abdulrahman Bin Faisal University, \\ Dammam, Saudi Arabia; 'Department of General Surgery, King Fahd Hospital of the \\ University, Imam Abdulrahman Bin Faisal University, Al-Khobar, Saudi Arabia; \\ dDepartment of Allergy and Immunology, University of Missouri Kansas City School of \\ Medicine, Kansas City, MO, USA
}

\section{Keywords \\ Erythema nodosum $\cdot$ Salmonella $\cdot$ Abscess}

\begin{abstract}
Erythema nodosum is a delayed-type hypersensitivity reaction with an unknown trigger in the majority of cases. It is characterized by the development of erythematous tender nodules on the shins. Septal panniculitis without vasculitis is a characteristic histopathological finding. We report the case of a 26-year-old woman who presented with a four-day history of an erythematous swollen left lower limb. She was treated with intravenous clindamycin for suspected cellulitis. However, her symptoms persisted. Punch biopsy revealed findings consistent with erythema nodosum. Two days later, she developed colicky abdominal pain associated with nonbloody diarrhea. Stool culture yielded Salmonella enterica serotype enteritidis. Two days after discharge, she presented again with a right breast abscess for which she underwent incision
\end{abstract}




\section{Case Reports in Gastroenterology}

Case Rep Gastroenterol 2019;13:456-461

DOI: 10.1159/000503894

(c) 2019 The Author(s). Published by S. Karger AG, Base www.karger.com/crg

Hassan et al.: Erythema Nodosum: A Manifestation of Salmonella Infection

and drainage along with antibiotic therapy. After discharge, she was symptom-free with complete resolution of the cutaneous lesions. The presented case is unique as it had multiple clinical manifestations of Salmonella infection including erythema nodosum, diarrhea, and presumably a breast abscess. It should be kept in mind that gastrointestinal symptoms are not necessarily the initial presentations of Salmonella infection.

\section{Introduction}

Infection with Salmonella species, gram-negative facultative anaerobic bacilli, is associated with significant morbidity and mortality worldwide. Diarrhea is the main clinical manifestation, but a wide spectrum of clinical presentations can be seen with such infections. Extraintestinal manifestations can include bacteremia, endocarditis, septic arthritis, and osteomyelitis.

Erythema nodosum is a skin condition characterized by tender and slightly raised erythematous subcutaneous eruptions predominantly localized in the pre-tibial areas [1]. It is the most common form of septal panniculitis (inflammation of the subcutaneous fat tissue) without primary vasculitis. It results from delayed hypersensitivity reactions to a wide range of antigens and triggers. The association between erythema nodosum and Salmonella infection is rare with few cases reported in the literature [2]. Herein, we describe the case of a young woman with erythema nodosum, in whom the gastrointestinal symptoms were not observed initially. The case demonstrated various clinical manifestations, including diarrhea, erythema nodosum, and breast abscess, presumably attributed to Salmonella enteritidis infection.

\section{Case Presentation}

A 26-year-old Filipino woman presented to the Emergency Department (ED) with a swollen erythematous left lower limb and ankles that had been painful upon movement for 4 days. These symptoms were associated with decreased appetite, general malaise, and headache. There was no history of fever, trauma, or travel preceding the onset of symptoms. She worked as a domestic helper, did not take any medication on a daily basis, and had unremarkable medical, surgical, family, and social history. One day prior to presentation, she was seen at a private clinic where she was diagnosed with a possible allergic reaction to an insect bite. She was sent home with oral analgesics. However, her symptoms persisted which prompted the ED visit.

Physical examination of the lower extremities revealed multiple raised erythematous lesions scattered over the surface of her left lower limb (Fig. 1). Bilateral inflamed ankles with pitting edema and slight pain on pressure were noted. The superficial lymph nodes were not palpable. Chest and abdominal examinations were unremarkable. The initial workup revealed: complete blood count with a leukocyte count of $11 \times 10^{3} / \mu \mathrm{L}$, hemoglobin of $13 \mathrm{~g} / \mathrm{dL}$, and platelet count of $395 \times 10^{3} / \mu \mathrm{L}$, normal serum comprehensive metabolic panel, erythrocyte sedimentation rate of $96 \mathrm{~mm} / \mathrm{h}$, and C-reactive protein of $3.2 \mathrm{mg} / \mathrm{dL}$. Lower limb ultrasonography revealed subcutaneous edema with no loculated fluid collection. 


\section{Case Reports in Gastroenterology}

Case Rep Gastroenterol 2019;13:456-461

DOI: $10.1159 / 000503894$

(c) 2019 The Author(s). Published by S. Karger AG, Basel www.karger.com/crg

Hassan et al.: Erythema Nodosum: A Manifestation of Salmonella Infection

The initial clinical impression was early stage cellulitis of the left lower limb. Therefore, she was administered intravenous clindamycin $(600 \mathrm{mg})$ every $8 \mathrm{~h}$, in addition to analgesics. Two days later, she developed colicky abdominal pain that was associated with watery nonbloody diarrhea. Stool samples were sent for Clostridium difficile toxin assay, microscopic examination, and culture. Salmonella enteritidis was identified in the culture (Fig. 2), the other tests revealed no abnormalities. Treatment began with the administration of oral ciprofloxacin (500 mg) every $12 \mathrm{~h}$, based on antibiotic susceptibility test results (Table 1 ).

The skin lesions did not improve following administration of ciprofloxacin. Therefore, the dermatology team was consulted, and a punch biopsy was performed. The histopathological examination of the biopsy specimen revealed septal thickening in the subcutaneous tissue with chronic inflammatory cellular infiltration and multinucleated giant cells consistent with erythema nodosum. The patient underwent further investigations, including a chest radiography, throat swab culture, tuberculin skin test, angiotensin-converting enzyme level, antistreptolysin 0 titer, and anti-nuclear antibody, all of which were unremarkable.

The patient showed clinical improvement with antibiotic therapy and supportive care; the diarrhea resolved, and ankle pain subsided. However, no remarkable changes were noted in the skin lesions. The patient was discharged to continue with oral ciprofloxacin treatment along with outpatient clinic follow-up visits.

Three days after discharge, the patient returned to the ED with right breast pain and swelling consistent with a breast abscess. She reported to having had this problem since the previous admission but had been reluctant to mention it at the time because her symptoms had been mild. The patient was not lactating and had no prior breast abscesses. After incision and drainage had been performed, a sample was sent for culture and sensitivity. The final culture result was negative, which was attributed to antibiotic use. She was followed up one month later at the clinic, at which point all of her symptoms had completely resolved.

\section{Discussion}

The classical manifestation of erythema nodosum is an acute eruption of tender, erythematous, warm nodules that are frequently located on the shins, ankles, and knees. These nodules usually develop over several days, with a prodrome of fever, malaise, and/or arthralgia [1]. The lesions may initially be bright red in color and be mistaken for cellulitis, similar to our case, particularly when the typical nodules are not apparent [3]. Ulceration is not seen, and the lesions resolve without scarring or atrophy. Non-steroidal anti-inflammatory drugs along with bedrest and supportive care are the main treatments for erythema nodosum with complete resolution expected within six weeks [1].

Despite being idiopathic in about half of all cases, erythema nodosum has been associated with numerous conditions. These conditions include infections, reactions to medications, inflammatory bowel disease, malignancy, sarcoidosis, and pregnancy. Infection is the most common identifiable trigger. Group A $\beta$-hemolytic streptococcal infection is by far the most frequently implicated bacterial infection [1]. Tuberculosis is another infection to be considered, particularly in endemic areas [1, 4]; however, this is becoming a less frequent cause [5]. Various other bacterial species have also been reported in association with this condition. 
Salmonella, particularly serotype enteritidis, is among the rare infectious causes of erythema nodosum with only a few cases reported in the medical literature [2]. The first case of erythema nodosum caused by Salmonella enteritidis was reported by Grossman and Katz [6] in 1984, in which the erythematous nodules appeared about one and half weeks after the onset of diarrhea in a young woman. Thereafter, a few cases have been reported, including those in a retrospective study conducted by Sota Busselo et al. [7], which showed that among 45 cases of erythema nodosum, 7 were caused by Salmonella enteritidis. Our case is novel in that the onset of erythema nodosum preceded the onset of diarrhea, unlike in previously reported cases [2]. The present case emphasizes that gastrointestinal symptoms are not always the first manifestation of Salmonella infection. In fact, it has been reported in the literature that systemic manifestations of Salmonella infection might occur without gastrointestinal manifestations [8].

Although pus culture obtained from the breast abscess was sterile, it might have been caused by Salmonella infection considering the time of presentation and lack of other risk factors or prior history of breast abscess. Breast abscesses caused by Salmonella enteritidis is a rare occurrence [9]. A previous report describes a case in which the patient presented with erythema nodosum and breast abscess owing to Salmonella enterica serotype poona [8]. Our case is unique in that it demonstrated various clinical manifestations, including diarrhea, erythema nodosum, and breast abscess, presumably attributed to Salmonella infection.

In conclusion, Salmonella infection might have variable clinical manifestations, and it must be noted that gastrointestinal symptoms might not be seen at the time of presentation. This case demonstrated a rare association of erythema nodosum and presumably breast abscess with Salmonella enteritidis.

\section{Statement of Ethics}

Written informed consent was obtained from the patient for the publication of this case report and accompanying images.

\section{Disclosure Statement}

The authors declare no conflicts of interest.

\section{Funding Sources}

This work did not receive any specific grant from funding agencies.

\section{Author Contributions}

A.H. and A.A. drafted the manuscript. M.A.S. and M.S. reviewed the literature. H.A. and S.A. edited the manuscript. All authors read and approved the final manuscript. 


\section{Case Reports in Gastroenterology}

\begin{tabular}{l|l}
\hline Case Rep Gastroenterol 2019;13:456-461 \\
\hline DOI: 10.1159/000503894 & $\begin{array}{l}\text { @ 2019 The Author(s). Published by S. Karger AG, Basel } \\
\text { www.karger.com/crg }\end{array}$ \\
\hline
\end{tabular}

Hassan et al.: Erythema Nodosum: A Manifestation of Salmonella Infection

\section{References}

1 Requena L, Requena C. Erythema nodosum. Dermatol Online J. 2002 Jun;8(1):4.

2 Haider S, Brar I, Mateo NB. Erythema nodosum and Salmonella enteritidis: a rare clinical association. Infect Dis Clin Pract. 2000;9(5):223-4.

3 Sawada T, Suehiro M, Takaya K. Lower-leg cellulitis-like manifestations of erythema nodosum induced by Chlamydophila pneumoniae infection. Indian J Dermatol. 2016 Mar-Apr;61(2):237.

4 Bjorn-Mortensen K, Ladefoged K, Simonsen J, Michelsen SW, Sørensen HC, Koch A, et al. Erythema nodosum and the risk of tuberculosis in a high incidence setting. Int J Circumpolar Health. 2016 Oct;75(1):32666.

5 Rizvi Z, Iqbal T, Javed A, Rizvi A. Erythema nodosum: a consequence of tuberculosis. Cureus. 2019 May;11(5):e4724.

6 Grossman ME, Katz B. Salmonella enteritidis enterocolitis: another cause of diarrhea and erythema nodosum. Cutis. 1984 Oct;34(4):402-3.

7 Sota Busselo I, Oñate Vergara E, Pérez-Yarza EG, López Palma F, Ruiz Benito A, Albisu Andrade Y. [Erythema nodosum: etiological changes in the last two decades]. An Pediatr (Barc). 2004 Nov;61(5):403-7.

$8 \mathrm{Al}$ Benwan K, Al Mulla A, Izumiya H, Albert MJ. Erythema nodosum and bilateral breast abscesses due to Salmonella enterica serotype Poona. J Clin Microbiol. 2010 Oct;48(10):3786-7.

9 Al-Ishaq Z, Sikdar O, Dobie D, Sircar T: Salmonella Enteritidis breast abscess: an unusual cause of breast abscess in the UK. BMJ Case Rep 2018 Oct;2018: pii: bcr-2018-226075.

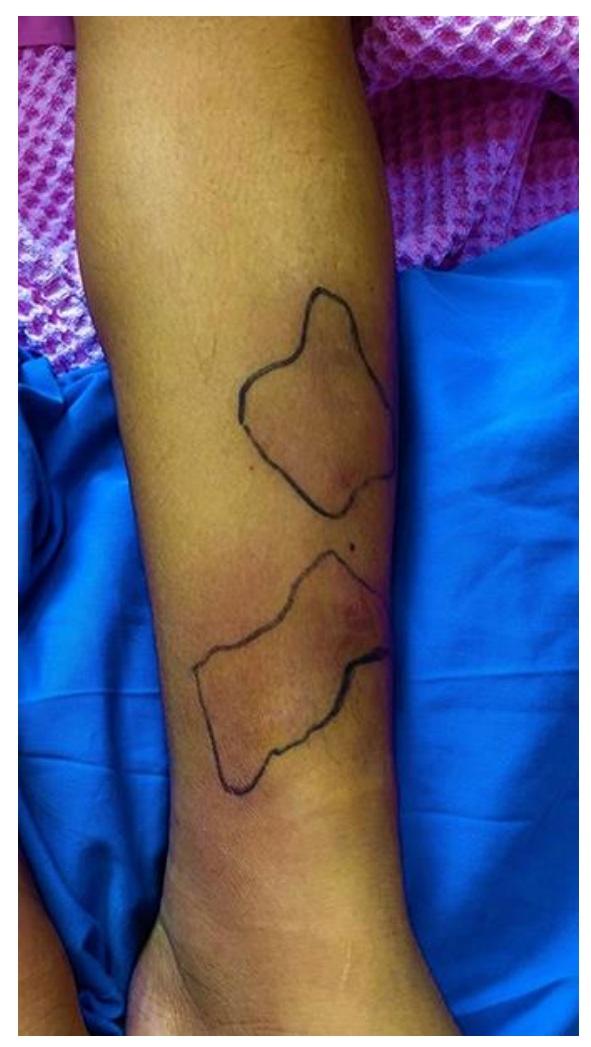

Fig. 1. A photograph of the patient's left leg 2 days after admission showing areas of erythema. 


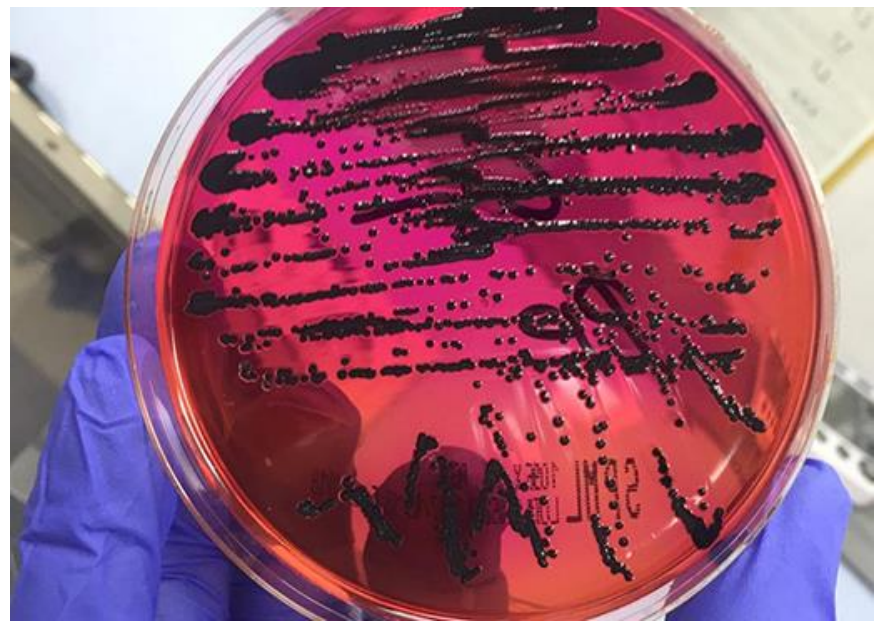

Fig. 2. Xylose-lysine deoxycholate agar showing black colonies of Salmonella enteritidis.

Table 1. Antibiotic susceptibility test of the isolated Salmonella enteritidis

\begin{tabular}{llll}
\hline Sensitive & \multicolumn{2}{c}{ Resistant } \\
\cline { 1 - 2 } Drug & MIC & \\
\hline Ampicillin & $\leq 2$ & Cefalotin \\
Amoxicillin/Clavulanic acid & $\leq 2$ & Cefoxitin \\
Piperacillin/Tazobactam & $\leq 4$ & Amikacin \\
Ceftazidime & $\leq 1$ & Gentamicin \\
Ceftriaxone & $\leq 1$ & Nitrofurantoin \\
Cefepime & $\leq 1$ & \\
Imipenem & $\leq 0.25$ & \\
Ciprofloxacin & $\leq 0.25$ & \\
Tigecycline & $\leq 0.5$ & \\
\hline
\end{tabular}

